\title{
The treatment of diabetic foot infections: focus on ertapenem
}

This article was published in the following Dove Press journal:

Vascular Health and Risk Management

5 November 2009

Number of times this article has been viewed

\author{
Michael Edmonds \\ Diabetic Foot Clinic, King's College \\ Hospital, Denmark Hill, London, UK
}

\begin{abstract}
Clinically, 3 distinct stages of diabetic foot infection may be recognized: localized infection, spreading infection and severe infection. Each of these presentations may be complicated by osteomyelitis. Infection can be caused by Gram-positive aerobic, and Gramnegative aerobic and anaerobic bacteria, singly or in combination. The underlying principles are to diagnose infection, culture the bacteria responsible and treat aggressively with antibiotic therapy. Localized infections with limited cellulitis can generally be treated with oral antibiotics on an outpatient basis. Spreading infection should be treated with systemic antibiotics. Severe deep infections need urgent admission to hospital for wide-spectrum intravenous antibiotics. Clinical and microbiological response rates have been similar in trials of various antibiotics and no single agent or combination has emerged as most effective. Recently, clinical and microbiological outcomes for patients treated with ertapenem were equivalent to those for patients treated with piperacillin/tazobactam. It is also important to judge the need for debridement and surgery, to assess the arterial supply to the foot and consider revascularization either by angioplasty or bypass if the foot is ischemic. It is also important to achieve metabolic control. Thus infection in the diabetic foot needs full multidisciplinary treatment.
\end{abstract}

Keywords: diabetes, foot, infection, antibiotics, ertapenem

\section{Introduction}

At some time in their life, $15 \%$ of people with diabetes develop foot ulcers. Eighty-five percent of amputations are preceded by an ulcer ${ }^{1}$ and there is an amputation every 30 seconds throughout the world. ${ }^{2}$ The main reason for this is that foot ulcers are highly susceptible to infection. ${ }^{3}$ This may spread rapidly leading to overwhelming tissue destruction and the need for amputation. Guidelines on the Diagnosis and Treatment of Diabetic Foot Infections have been issued from the Infectious Diseases Society of America (IDSA) ${ }^{4}$ and also by The International Working Group on the Diabetic Foot, which produced its International Consensus Guidelines on Diagnosing and Treating Infected Diabetic Foot in $2003^{5}$ and recently guidelines for the treatment of Diabetic Foot Osteomyelitis in 2007. ${ }^{6,7}$ New developments have recently been reviewed by Lipsky. ${ }^{8}$

\section{Classification of infection}

Various classifications of infection exist and the ISDA has staged infection from mild to moderate to severe. ${ }^{4}$ Mild infection is characterized by the presence of 2 manifestations of inflammation (purulence, or erythema, pain, tenderness, warmth or induration), with cellulitis/erythema extending less than $2 \mathrm{~cm}$ around the ulcer, and infection is
Correspondence: Michael Edmonds Diabetic Foot Clinic, King's College Hospital, Denmark Hill, London, UK Email michael.edmonds@kch.nhs.uk 
limited to the skin or superficial subcutaneous tissue with no other local complications or systemic illness. In moderate infection, the patient has one of the following characteristics: cellulitis extending $>2 \mathrm{~cm}$, lymphangitic streaking, spread beneath the superficial fascia, deep-tissue abscess, gangrene, and involvement of muscle, tendon, joint or bone, but is systemically well and metabolically stable. In severe infection, the patient has systemic toxicity or metabolic instability (eg, fever, chills, tachycardia, hypotension, confusion, vomiting, leukocytosis, acidosis, severe hyperglycemia, or azotemia). Validation of the IDSAs diabetic foot infection classification system has been reported in a longitudinal study of 1666 persons with diabetes. ${ }^{9}$ There was an observed trend toward an increased risk for amputation, higher-level amputation and lower extremity-related hospitalization with increasing infection severity. Other classifications include limb threatening and non-limb threatening infections. ${ }^{10}$

\section{Clinical presentation of infection}

Clinically, 3 distinct stages of diabetic foot infection may be recognized: localized infection, spreading infection and severe infection. Each of these presentations may be complicated by osteomyelitis. ${ }^{11}$

\section{Localized infection}

Localized infection refers to infection in the ulcer bed or immediately surrounding skin. This may present with

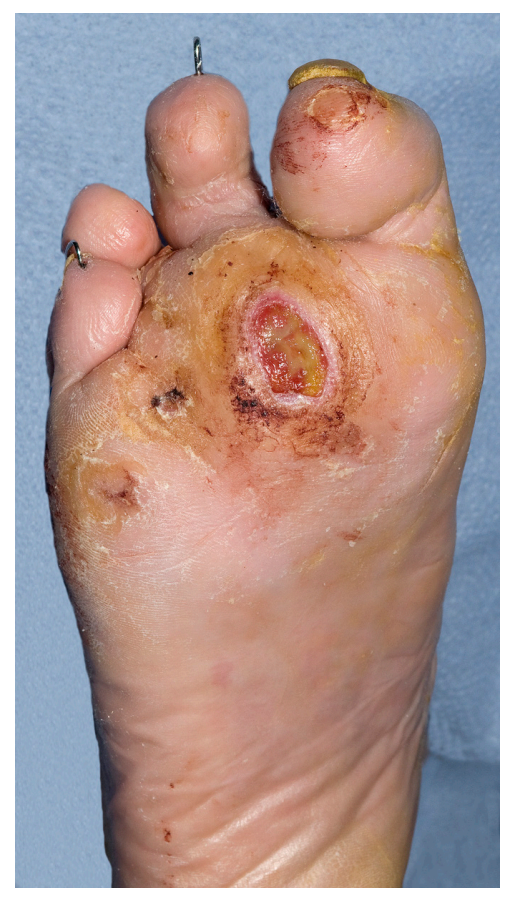

Figure I Infection in ulcer bed with mild surrounding erythema. purulent discharge and surrounding erythema, but often the classical signs of infection are diminished by the presence of neuropathy and ischemia (Figure 1). Thus the signs of infection may be very subtle. Galen's classical signs and symptoms of redness, heat, pain and loss of function may not be evident. However, early warning signs of infection and signs of deterioration should be searched for with great assiduity in all diabetic foot patients, especially those with breaks in the skin.

\section{Spreading infection}

Sepsis has progressed to give signs of spreading infection emanating from the ulcer such as diffuse spreading erythema, edema, lymphangitis and lymphadenitis, and in addition, there will usually be local signs of infection as described above (Figure 2). Systemic symptoms and signs may be present when the foot has extensive diffuse cellulitis, The portal of entry of infection may be a corn, callus, blister, fissure or any other skin break.

\section{Severe infection}

This refers to ulcers with extensive deep soft tissue infection. In the presence of neuropathy, pain and throbbing may be absent, but if present this is a danger sign, usually indicating serious infection with pus within the tissues. Palpation may reveal fluctuance, suggesting abscess formation. There may be bulging of the plantar surface of the foot. Discrete abscesses are relatively uncommon in the infected diabetic foot. Often there is a generalized sloughing of the ulcer and surrounding subcutaneous tissues which eventually liquefy and disintegrate. In late infection, there is swelling and a brawny effect. The tissues may be sloughing and breaking down and blistered, or fluctuant, and often never recover but need

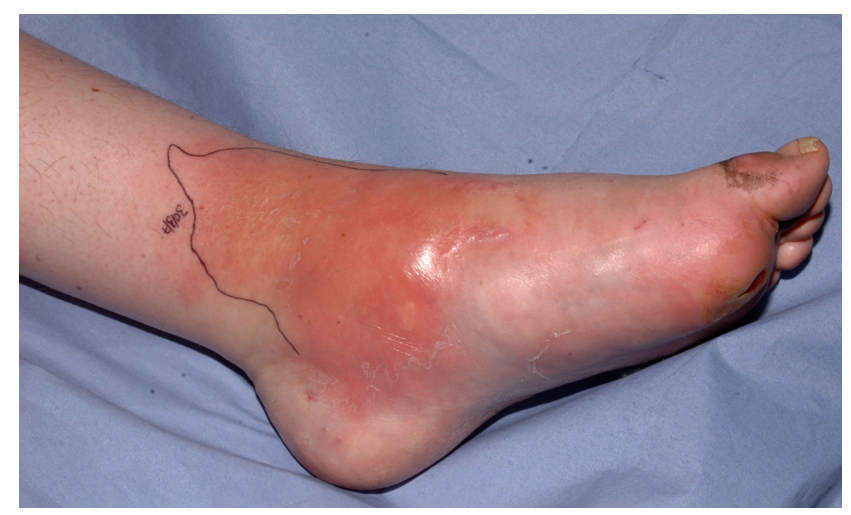

Figure 2 Spreading cellulitis. 
surgical removal. Severe infection can also present as a bluish-purple discoloration when there is inadequate supply of oxygen to the soft tissues leading to gangrene (Figure 3). This is caused by increased metabolic demands of infection and a reduction of blood flow to the skin, secondary to a septic vasculitis of the cutaneous circulation. Purple blebs may indicate subcutaneous necrosis. Blue discoloration can occur in both the neuropathic and also the neuroischemic foot, particularly in the toes, and in the neuroischemic foot must not be automatically attributed to worsening ischemia.

This stage may also be associated with septicemia, with the patient presenting with hypotension and organ failure. Signs of severe infection may include drowsiness shivering, tachycardia, hypotension, reduced body temperature $(<35$ $\left.{ }^{\circ} \mathrm{C}\right)$ or high fever $\left(>40{ }^{\circ} \mathrm{C}\right)$. However, systemic signs and symptoms are notoriously absent in many severe infections of the diabetic foot. Among patients hospitalized for late infections only $12 \%$ to $35 \%$ have significant fever and only $50 \%$ of episodes of severe cellulitis will provoke a fever or leucocytosis. However, when fever is present it usually indicates a severe infection and the deep spaces of the foot are usually involved with tissue necrosis, severe cellulitis and possible bacteremia.

\section{Osteomyelitis}

Usually osteomyelitis will present in association with ulceration and soft tissue infection. Clinically, osteomyelitis may be suspected when a sterile probe inserted into the base of the ulcer penetrates to bone. This may happen in an

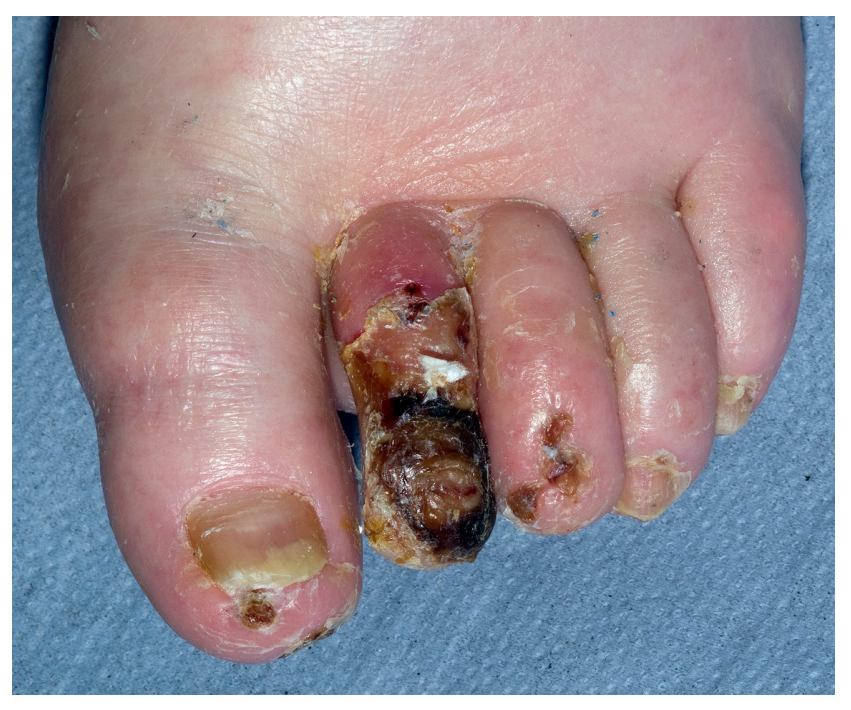

Figure 3 Infective necrosis of second toe. apparently clean, uninfected ulcer, but osteomyelitis must still be suspected. It may present as obvious fragmentation of the bone within the ulcer bed, which is easily visible. Chronic osteomyelitis of a toe has a swollen, red, sausage-like appearance. It is most commonly diagnosed on X-ray. Loss of cortex, fragmentation and bony destruction on X-ray are signs of osteomyelitis. These changes may take 10 to 14 days to develop. MRI is the best imaging technique to diagnose osteomyelitis and can demonstrate edema and abscesses in bone. However bone edema may also be present in Charcot's osteoarthropathy. Osteomyelitis may be confirmed in theory by a positive bone culture or bone biopsy showing bone death, inflammation and repair. Bone biopsy is often not very practical, and then the diagnosis is made on clinical and radiological grounds.

\section{Microbiology of the diabetic foot}

The microbiology of the diabetic foot is unique. Infection can be caused by Gram-positive aerobic, Gram-negative aerobic and anaerobic bacteria, singly or in combination. As there may be a poor immune response of the diabetic patient, even bacteria normally regarded as skin commensals may cause severe tissue damage. Furthermore, when Gram-negative bacteria such as Citrobacter, Serratia, Pseudomonas and Acinetobacter are isolated from a deep ulcer swab or curettings they should not, be regarded as automatically insignificant. Severe infections are often polymicrobial and both Gram-positive and Gram-negative organisms are present together with anaerobes. Severe subcutaneous infection by Gram-negative and anaerobic organisms produces gas which may be detected by palpating crepitus on the lower limb and can be seen on X-ray. The presence of gas does not automatically mean that the classical gas gangrene organism Clostridium perfringens is present. The most common cause is a synergism of Gram-negative organisms and anaerobes although Staphylococcus aureus can also lead to non-clostridial necrosis.

In a recent review of the microbiology of 427 positive cultures from moderate to severe infections, ${ }^{12}$ infections were typically polymicrobial, and almost half included anaerobes; $84 \%$ were polymicrobial, $48 \%$ grew only aerobes, $43.7 \%$ had both aerobes and anaerobes, and $1.3 \%$ had only anaerobes. Cultures yielded an average of 2.7 organisms per culture (range 1 to 8 ) for aerobes and 2.3 organisms per culture (range 1 to 9 ) for anaerobes. The main aerobic organisms were oxacillin-susceptible $S$. aureus (14.3\%), oxacillin-resistant $S$. aureus (4.4\%), coagulase-negative Staphylococcus spp. (15.3\%), Streptococcus spp. (15.5\%), 
Enterococcus spp. (13.5\%), Corynebacterium spp. (10.1\%), members of the family Enterobacteriaceae (12.8\%), and Pseudomonas aeruginosa (3.5\%). The predominant anaerobes were Gram-positive cocci (45.2\%), Prevotella spp. (13.6\%), Porphyromonas spp. (11.3\%), and the Bacteroides fragilis group (10.2\%).

It is important to have a working knowledge of the principal bacteria and their local antibiotic sensitivities, including awareness of the prevalence of resistant organisms. However, in every patient, individual sensitivities of each organism isolated on culture should be sought to guide rational antibiotic therapy. When the patient initially presents, microbiology data are not available and empirical therapy will need to be started and there should be close co-operation between the microbiology laboratory and the diabetic foot service.

\section{S. aureus}

S. aureus is the commonest pathogen in the diabetic foot and flucloxacillin is the ideal treatment. Ertythromycin and clarithromycin can also be used. Clindamycin is usually effective but it is necessary to be aware of antibiotic-induced colitis especially in the elderly and postoperative patients. Rifampicin and fucidin are also good antistaphylococcal agents, but they should not be given alone as resistance will develop rapidly. They should each be accompanied by a further antistaphylococcal agent.

\section{Methicillin-resistant S. aureus (MRSA)}

MRSA is associated with the whole spectrum of clinical presentations of diabetic foot infections and commonly occurs in patients who have been in hospital. It can be simply a commensal with no signs of invasive infection but it can also cause severe infections, osteomyelitis and bacteremia. Since colonization precedes infection, contact with other patients with MRSA is a risk factor. Other risk factors include repeated hospitalizations, lengthy hospital stays, prior antimicrobial therapy and the presence of surgical wounds. The frequency of MRSA infections is increasing in the diabetic foot. ${ }^{13}$ In a study investigating the prevalence of MRSA in infected and uninfected diabetic foot ulcers of 84 patients with diabetes, $S$. aureus was the most common pathogen among the Gram-positive bacteria isolated from ulcers, and almost $50 \%$ of $S$. aureus isolates were MRSA. The prevalence of MRSA was significantly higher in patients with infected foot ulcers. ${ }^{14}$ However, MRSA infections are not necessarily more pathogenic than conventional $S$. aureus infections. They do frequently cause more extensive tissue destruction because they are often not diagnosed until late. This is an important reason to maintain frequent bacteriological surveillance on all ulcers in diabetic feet. Hospital acquired MRSA is multiresistant to all beta lactams antibiotics and to a varying extent macrolides, fluoroquinolones and aminoglycosides, but are usually sensitive to gentamicin.

MRSA may be also be acquired in the community. ${ }^{15}$ This has been associated with outbreaks in groups of individuals with close contact in institutions such as prisons which can then be transferred to hospitals. These MRSA do not have the multiresistance of the hospital acquired MRSA but nevertheless can rapidly progress to severe infections. Approximately two-thirds possess the Panton-Valentine leukocidin toxin, which acts to form pores in the cell membrane of mononuclear cells and polymorphonuclear cells and can lead to severe tissue necrosis.

MRSA can lead to invasive infection and in these circumstances it is best to give vancomycin intravenously with dosage to be adjusted according to serum levels, or teicoplanin. These antibiotics may need to be accompanied by either sodium fusidate or rifampicin orally. New antimicrobial agents have become available. ${ }^{16}$ Linezolid is active against MRSA and has good soft tissue and bony penetration. ${ }^{17,18}$ It is well absorbed. It is necessary, however, to check the platelet count regularly as there may be some marrow suppression with this antibiotic. Courses should not exceed 28 days. The combination of antibiotics quinupristin and dalfopristin can be used when an MRSA infection has not responded to other antibacterials. Daptomycin and tigecycline may also be used in MRSA infections. ${ }^{19}$ MRSA can also be treated with clindamycin but sensitivity needs to be confirmed as MRSA resistance to clindamycin has emerged. If MRSA is isolated in localized infections, oral therapy can be given with two of the following: sodium fusidate $500 \mathrm{mg}$ tds, rifampicin $300 \mathrm{mg}$ tds, trimethoprim $200 \mathrm{mg}$ bd or doxycycline $100 \mathrm{mg}$ daily, according to sensitivities.

\section{Streptococcus group A, B, C, E, F and G}

Streptococcus group B is being increasingly recognized as an important pathogen in the diabetic foot although C, E, F and $\mathrm{G}$ can also infect the foot. ${ }^{20}$ Streptococcus group A rarely causes infection but when it does, it causes a severe blistering cellulitis and tissue destruction. The Streptococcus milleri group of organisms are also beta hemolytic streptococci that can cause abcesses in the foot. Streptococci can be treated with amoxicillin. Clindamycin, rifampicin and erythromycin may also be also active against streptococci. 


\section{Enterococcus}

Enterococcus faecalis is rarely pathogenic. It may be selected out by cephalosporin treatment. If it is causing definite infection then it may be treated with amoxicillin or vancomycin. Vancomycin-resistant enterococcus (VRE) is a problem with hospitalized patients previously treated with antibiotics (especially patients in renal failure). It is necessary to assess whether it is actually causing tissue destruction as it is normally not pathogenic. However, in the immunocompromised diabetic patient, especially in the ischemic foot, it may be responsible for infection and should therefore be treated with appropriate antibiotics as suggested by culture sensitivities. Enterococcus faecium may need vancomycin treatment and quinupristin/dalfopristin and linezolid are useful for vancomycin-resistant $E$. faecium infections.

\section{C. perfringens}

C. perfringens is a Gram-positive bacillus that can produce many toxins including including alpha-toxin. It is well known for causing clostridial myonecrosis or gas gangrene, although this is more often caused by non-clostridial organisms, namely Gram-negatives and anaerobes.

\section{Anaerobes}

These are commonly found in deep infections but anaerobes are also a feature of many chronic wounds even when they are superficial. They are also associated with necrotic wounds. Anaerobes can act synergistically with Gram-positive and Gram-negative aerobes to cause severe tissue destruction as well as synergistic and gas gangrene. The latter is traditionally associated with $C$. perfringens but is now usually caused by synergy between Gram-negative organisms and anaerobes.

Metronidazole is the treatment of choice. Clindamycin and co-amoxiclav (amoxicillin/clavulanic acid) also have antianaerobic activity. Meropenem, piperacillin/tazobactam and ertapenem are also active against anaerobes. Gas in the soft tissues of an ill diabetic patient presenting with cellulitis raises the serious possibility of synergistic gangrene. In such circumstances, clindamycin would be preferable to flucloxacillin because of its effect on protein, and hence toxin production.

\section{Enterobacteriaceae}

The Enterobacteriaceae, which include Klebsiella, Escherichia coli, Proteus, Enterobacter, Citrobacter, Serratia and other Gram-negative bacteria, can be definitely pathogenic in the diabetic foot especially when they are in a pure growth or as part of a polymicrobial deep infection. Oral agents that are available to treat these Gram-negatives are ciprofloxacin and other quinolones and trimethoprim. Parenteral agents include ceftazidime, aminoglycosides, meropenem, piperacillin/tazobactam, ticarcillin/clavulanate, tigecycline and ertapemem. It is crucial to obtain sensitivity patterns with Gram negative organisms and not depend on empirical therapy alone.

Recently, Gram-negative bacteria have acquired various resistance patterns through the development of certain enzymes and this is relevant to the choice of antibiotic therapy. Organisms have developed extended spectrum beta-lactamases which are known as ESBLs. By this means, they have developed resistance to extended-spectrum (third generation) cephalosporins (eg, ceftazidime, cefotaxime, ceftriaxone) but not to carbapenems (eg, meropenem or ertapenem). ESBL enzymes are most commonly produced by E. coli, Klebsiella pneumoniae and Enterobacter aerogenes. Another group of lactamases are AmpC beta-lactamases which are typically encoded on the chromosomes of many Gram-negative bacteria including Citrobacter, Serratia and Enterobacter spp. where expression is usually inducible. Thus, organisms considered susceptible by in vitro testing can become resistant during treatment with cephalosporins. Carbapenems are the only reliable beta-lactam drugs for the treatment of severe Enterobacter infections.

\section{Pseudomonas}

There are many members of the genus Pseudomonas, and $P$. aeruginosa is an important human opportunist bacterium in the diabetic foot. It can be responsible for a spectrum of presentations from superficial colonization of ulcers to extensive tissue damage, including osteomyelitis, septic arthritis and bacteremia. It may be sensitive to ciprofloxacin as an oral agent. Otherwise parenteral therapy is necessary and includes ceftazidime, aminoglycosides, meropenem, piperacillin/tazobactam, and ticarcillin/clavulanate.

\section{Stenotrophomonas maltophilia}

Stenotrophomonas maltophilia is the type species of Stenotrophomonas. This Gram-negative bacterium was initially classified as Pseudomonas maltophilia. It is resistant to many broad-spectrum antibiotics (including all carbapenems), and is usually resistant to aminoglycosides, antipseudomonal penicillins and third-generation cephalosporins. It is often acts as a colonizer. However, if isolated from a purulent wound, S. maltophilia may be the cause of the patient's wound infection. Many strains of 
S. maltophilia are sensitive to co-trimoxazole, ticarcillin/ clavulanate and colomycin.

\section{Treatment}

The development of infection constitutes a foot care emergency, which requires referral to specialized foot-care team within 24 hours. The underlying principles are to diagnose infection, culture the bacteria responsible, treat aggressively with antibiotic therapy and consider the need for debridement and surgery. Usually, therapy is commenced with wide spectrum therapy which is then focused according to the microbiology culture results. It is also important to assess the arterial supply to the foot and consider revascularization either by angioplasty or bypass if the foot is ischemic. It is also important to achieve metabolic control. Thus infection in the diabetic foot needs full multidisciplinary treatment. The team managing these infections should preferably include, or have ready access to, an infectious diseases specialist or a medical microbiologist.

When managing these very difficult and unstable feet, decision making should be guided by symptoms and signs of infection, results of properly taken wound swabs and tissue cultures and past and present knowledge of individual patients. While devising a management plan for the different presentations of infection it is important to address these questions: Does the patient need oral or systemic antibiotics? Does the patient need surgery? However, evidence from systematic reviews to inform these decisions is limited..$^{21,22}$

In a systematic review of the evidence for antimicrobial interventions for foot ulcers in diabetes, 23 studies were assessed for the effectiveness or cost-effectiveness of antimicrobial agents: intravenous antibiotics $(\mathrm{n}=8)$; oral antibiotics $(n=5)$; topical antimicrobials $(n=4)$; subcutaneous granulocyte-colony stimulating factor $(\mathrm{G}-\mathrm{CSF})(\mathrm{n}=4)$; ayurvedic preparations $(\mathrm{n}=1)$ : and sugar vs antibiotics vs standard care $(n=1)$. The review concluded that the trials were small and too dissimilar to be pooled. There was no strong evidence for any particular antimicrobial agent for the prevention of amputation, resolution of infection, or ulcer healing. Clinical clues to guide antibiotic selection have recently been reviewed. ${ }^{23}$ Initial antibiotic therapy for diabetic foot infections is usually empirical but several principles may aid to avoid selecting either an unnecessarily broad or inappropriately narrow regimen. Firstly, clinically severe infections require broad-spectrum therapy, while less severe infections may not. Second, aerobic Gram-positive cocci, particularly
S. aureus (including MRSA for patients at high-risk) should always be covered. Third, therapy should also be aimed at aerobic Gram-negative pathogens if the infection is chronic or has failed to respond to previous antibiotic therapy. Fourth, anti-anaerobic agents should be considered for necrotic or gangrenous infections on an ischemic limb.

\section{Localized infection}

Localized infections can generally be treated with oral antibiotics on an outpatient basis. Several antibiotics have been shown to be effective in clinical trials including cephalexin, clindamycin, ciprofloxacin, ofloxacin, levofloxacin, clindamycin, pexiganan and linezolid. ${ }^{24}$ However, no single drug or combination of agents appears to be better than others. Antibiotics should be consistent with local antibiotic policies and initially, commonly used first line antibiotics should be prescribed and new antibiotics reserved for later use for resistant organisms.

If MRSA is grown and there are no local or systemic signs of infection, topical mupirocin $2 \%$ ointment (if sensitive) may be used. If MRSA is grown and accompanied by local signs of infection, oral therapy with two of the following should be considered: sodium fusidate, rifampicin, trimethoprim and doxycycline, according to sensitivities, together with topical mupirocin $2 \%$ ointment.

\section{Spreading infection}

This condition should be treated with systemic antibiotics. It can sometimes be treated at the patient's home with intramuscular or intravenous antibiotics under close surveillance by relatives and frequent visits from the community nurse. In these circumstances, ceftriaxone may be given intramuscularly together with metronidazole orally. Ceftriaxone has been demonstrated to be just as efficacious as ticarcillin/ clavulanate. ${ }^{25}$ However, where the cellulitis is extensive, or in the ischemic foot, intravenous antibiotics and hospital admission will be needed at the outset for patients with spreading infection.

At initial presentation, it is important to prescribe a wide spectrum of antibiotics for three reasons:

a. it is impossible to predict the number and type of organisms from the clinical presentation

b. there is no way of predicting who will develop a rapidly ascending infection which becomes limb-threatening and even life-threatening

c. diabetic patients are immunosuppressed. The neuropathy and ischemia of the diabetic foot reduces the local 
resistance to invading bacteria. As is attributed to Louis Pasteur: "The germ is nothing. It is the terrain in which it grows that is everything."

\section{Severe infection}

Severe infections need urgent admission to hospital for wide spectrum intravenous antibiotics. Indications for urgent surgical intervention are infected sloughy tissue, localized fluctuance and expression of pus, crepitus with gas in the soft tissues on X-ray and purplish discoloration of the skin indicating subcutaneous necrosis. Infected tissue should be sent for culture after debridement. Clinical and microbiological response rates have been similar in trials of various antibiotics and no single agent or combination has emerged as most effective. Recently, clinical and microbiological outcomes for patients treated with ertapenem were equivalent to those for patients treated with piperacillin/ tazobactam, in a randomized, double-blinded, multicenter trial in adults $(n=586)$ with diabetes and a foot infection classified as moderate-to-severe and requiring intravenous antibiotics. ${ }^{26}$ For further discussion of the role of ertapenem and piperacillin/tazobactam see below.

It is important to have a practical approach to the treatment of severe infections reserving complex new antibiotics for resistant organisms, as described in the approach to mild infections. Ideally, the diabetic patient with severe cellulitis needs admission for intravenous antibiotics. If admission is not possible, then ceftriaxone may be given intramuscularly together with metronidazole orally. Ceftriaxone has been demonstrated to be just as efficacious as ticarcillin/ clavulanate. On review as an outpatient, if cellulitis is controlled, ceftriaxone intramuscularly and metronidazole orally should be continued and the patient reviewed one week later. If cellulitis is increasing, then the patient should be admitted for intravenous antibiotics. A standard broad spectrum, parenteral, quadruple antibiotic therapy (flucloxacillin, amoxicillin, metronidazole and ceftazidime) has been developed and used sucessfully at King's College Hospital, London for the last 15 years for the treatment of severe limb threatening infections in a variety of diabetic patients with varying levels of associated co-morbidity such as lower limb ischemia and/or renal impairment.

Quadruple therapy may be used including amoxycillin, flucloxacillin, metronidazole and ceftazidime. ${ }^{11}$ If the patient is allergic to penicillin, amoxycillin and flucloxacillin should be replaced with vancomycin (with doses adjusted according to serum levels). Furthermore, if the patient is known to have recent MRSA infection, then vancomycin should also be substituted for amoxicillin and flucloxacillin. On admission the foot should be urgently assessed as to the need for surgical debridement. On follow-up, the infected foot should inspected daily to gauge the initial response to antibiotic therapy. Appropriate antibiotics should be selected when sensitivities are available. If MRSA is isolated, then vancomycin (dosage to be adjusted according to serum levels) or teicoplanin should be given. These antibiotics may need to be accompanied by a further appropriate oral antibiotic such as sodium fusidate or rifampicin. When the signs of cellulitis have resolved intravenous antibiotic therapy can be changed to the appropriate oral therapy, usually any two of sodium fusidate, rifampicin trimethoprim or doxycycline.

\section{Osteomyelitis}

Osteomyelitis can complicate any of the above infective presentations.

\section{Initial treatment}

When diagnosis of osteomyelitis is made clinically, an empirical regime with good bone penetration should be given such as rifampicin $300 \mathrm{mg}$ tds and ciprofloxacin $500 \mathrm{mg}$ bd. On review, antibiotic selection is guided by the results of deep swabs tissue biopsy or bone culture. Some centers base their antibiotic selection on bone culture results alone. However, sinus tract or ulcer culture results may be helpful. A bone biopsy may be difficult to obtain and is not without risk in the neuroischemic foot. It is useful to choose antibiotics with good bone penetration, such as sodium fusidate $500 \mathrm{mg}$ tds, rifampicin $300 \mathrm{mg}$ tds, clindamycin $300 \mathrm{mg}$ tds and ciprofloxacin $500 \mathrm{mg}$ bd.

\section{Follow-up plan}

Oral antibiotics should be given for at least 12 weeks. Parenteral therapy has in the past been given for 4 to 6 weeks followed by oral therapy. However, it may be possible to limit the parenteral therapy to 2 weeks and follow this with appropriate oral antibiotics (if the infected bone is resected then a shorter course of antibiotics such as 4 weeks may be necessary). Conservative therapy is often successful, and is associated with resolution of cellulitis and healing of the ulcer.

\section{Antibiotics used mainly against Gram-positive organisms Amoxicillin}

This antibiotic is active against streptococci but is inactivated by penicillinases that are produced by $S$. aureus and by Gramnegative bacteria such as $E$. coli. 


\section{Co-amoxiclav}

This is a combination of amoxicillin and clavulanic acid. The latter is a beta-lactamase inhibitor, thus widening the spectrum of activity of co-amoxiclav against beta lactamase producing bacteria that are resistant to amoxicillin including staphylococci, anaerobes and Gram-negative bacteria. The risk of liver toxicity is 6 times greater with co-amoxiclav compared with amoxicillin.

\section{Flucloxacillin}

This antibiotic is not destroyed by pencillinases and thus it is effective against penicillin-resistant staphylococci. When given intravenously, its dosage may be increased to $2 \mathrm{~g}$ qds in staphylococcal bacteremia or osteomyelitis.

\section{Erythromycin and clarithromycin}

They have a similar spectrum to penicillin and are thus useful against staphylococci and streptococci in patients who are allergic to penicillin. There is an increased risk of myositis and rhabdomyolysis if the patient is on statin therapy. Thus, statin therapy may be stopped for the duration of erythromycin therapy. If the patient develops intolerance to erythromycin, particularly gastrointestinal side-effects, then clarithromycin may be used.

\section{Fucidin}

This is active against penicillin-resistant staphylococci. It has good bone penetration and is useful in osteomyelitis. Resistance to it develops quickly if it is given alone and therefore it should be given with another anti-staphylococcal agent. It is useful in combination therapy to treat MRSA infections. Liver function should be monitored if therapy is prolonged and it should be given with caution in patients with liver disease.

\section{Doxycycline}

This antibiotic can be used in treating MRSA infections. It should be used with caution in patients with hepatic impairment.

\section{Rifampicin}

This is active against staphylococci and streptococci and has good soft tissue and bone penetration. Patients should be warned that if they develop nausea, vomiting or malaise they should report this immediately as it may reflect liver dysfunction which is a well-described but rare side-effect of rifampicin therapy. It should be given with caution in patients with existing liver disease. Patients should be warned that their body secretions will turn red. Rifampicin should not be given alone because resistance can develop rapidly.

\section{Clindamycin}

This has very good soft tissue and bone penetration and is active against staphylococci, streptococci and anaerobes including B. fragilis. However, historically it has been linked with antibiotic associated colitis caused by Clostridium difficile infections although this can occur with many antibiotics.

\section{Vancomycin}

This is active against Gram-positive organisms and is usually used for MRSA infections. Blood levels should be monitored and trough levels should not be less than $15 \mathrm{mg} / \mathrm{L}$.

\section{Teicoplanin}

This is a glycopeptide antibiotic which is active against Gram-positive organisms including MRSA. It can be given intravenously but also intramuscularly. This is a convenient therapy to be given at home.

\section{Linezolid}

Linezolid is active against Gram-positive organisms, including MRSA and vancomycin-resistant enterococci. It can be given orally or intravenously. It may cause marrow suppression and regular platelet counts are advisable. It should not be given for more than 28 days. Linezolid has been shown to be superior to co-amoxiclav (amoxicillin/clavulanic acid) and ampicillin/sulbactam for diabetic foot infections with and without osteomyelitis. ${ }^{17}$ It has also been shown to be at least equivalent to vancomycin in treating soft tissue infections and superior to vancomycin where the pathogen is MRSA. ${ }^{27}$

\section{Daptomycin}

Daptomycin is a novel cyclic lipopeptide with activity against a large number of resistant Gram-positive pathogens including MRSA, VRE and glycopeptide intermediate-susceptible S. aureus (GISA). It is given intravenously and has good soft tissue penetration. Weekly creatine phosphokinase levels should be monitored. ${ }^{28,29}$ The clinical and microbiological efficacy and safety of daptomycin were similar to those of commonly used comparator antibiotics for treating infected diabetic foot ulcers caused by Gram-positive pathogens. This was demonstrated in a randomized, controlled trial comparing daptomycin with vancomycin or semi-synthetic penicillins for complicated skin and skin-structure infections. ${ }^{30}$

\section{Quinupristin/dalfopristin}

This is a combination of the two antibiotics quinupristin and dalfopristin which work synergistically against Gram-positive organisms including MRSA. 


\section{Trimethoprim}

It has reasonable soft tissue penetration and is active against Gram-positive and Gram-negative bacteria. It is also useful in combination therapy against MRSA.

\section{Tigecycline}

This is a glycylcycline antibiotic that is structurally similar to tetracycline antibiotics. Tigecycline was active against $83.7 \%$ of all the strains against bacterial strains isolated from diabetic foot infections, especially Gram-positive cocci (97.3\%), in particular MRSA (96\%), Enterobacteriaceae $(88.5 \%)$ and anaerobes (100\%). Exclusively Pseudomonas and Proteae were not covered by this antibiotic. ${ }^{31}$

\section{Antibiotics used mainly against Gram-negative organisms Ciprofloxacin}

This is a useful quinolone antibiotic active against Gram-negative organisms and has good soft tissue and bone penetration. It has only moderate activity against Gram-positive organisms. It is relatively well tolerated but occasionally can give neurological side-effects and can rarely predispose to hypoglycemia in certain patients. Other quinolones include levofloxacin and moxofloxacin. Moxifloxacin, a broad-spectrum fluoroquinolone which can be administered by either intravenous or oral routes. To assess the efficacy of moxifloxacin for treating diabetic foot infections, a subset of diabetic patients with these infections who were enrolled in a prospective, double-blind study that compared the efficacy of moxifloxacin with piperacillin/tazobactam and amoxicillin/clavulanate were analyzed. Intravenous \pm oral moxifloxacin was as effective as intravenous piperacillin/ tazobactam \pm amoxicillin/clavulanate. ${ }^{32}$

\section{Septrin}

This is a combination of trimethoprim and sulphmethoxazole and is occasionally used to treat resistant Gram-negative organisms such as Stenotrophomonas maltophilia but should only be used if other antibiotics against Gram-negative organisms are not appropriate.

\section{Ceftriaxone}

This is a useful antibiotic that can be given either intravenously or intramuscularly when it is administered as $1 \mathrm{~g}$ in $3.5 \mathrm{~mL}$ of $1 \%$ lignocaine. It needs to be given only once a day. This can be given in the community on a once daily basis. It has a wide spectrum of activity but is not active against MRSA or Pseudomonas.

\section{Ceftazidime}

This is useful as an initial agent to cover Gram-negative infections as it is usually active against Pseudomonas. If the dosage is not reduced in renal impairment, then the patient may develop muscular twitching and even fits. Penetration of ceftazidime into bone from severely ischemic limbs is satisfactory. ${ }^{33}$ Ten patients received $2000 \mathrm{mg}$ of ceftazidime intravenously before undergoing lower-extremity amputation for ischemia. Bone and plasma concentrations were determined by HPLC. The bone concentrations were corrected for blood contamination. In all but one sample ceftazidime was detectable. These data indicate that ceftazidime penetrates into bone of severely ischemic limbs.

\section{Piperacillin/tazobactam}

This antibiotic is given intravenously and has a wide spectrum of activity including Gram-positive and Gram-negative organisms including Pseudomonas and anaerobes. It may be useful against bacteria with extended spectrum betalactamases. In an open-label, randomized study comparing efficacy and safety of intravenous piperacillin/tazobactam $(\mathrm{P} / \mathrm{T})$ and ampicillin/sulbactam $(\mathrm{A} / \mathrm{S})$, clinical efficacy rates (cure or improvement) were statistically equivalent overall ( $81 \%$ for $\mathrm{P} / \mathrm{T}$ vs $83.1 \%$ for $\mathrm{A} / \mathrm{S}$ ), and median duration of treatment was similar in the clinically evaluable populations (9 days for $\mathrm{P} / \mathrm{T}, 10$ days for $\mathrm{A} / \mathrm{S}){ }^{34}$

\section{Ticarcillin/clavulanic acid}

This is given intravenously and is active against Pseudomonas, and other Gram-negative bacteria including Proteus spp. and B. fragilis.

\section{Imipenem with cilastin}

This is a carbapenem with broad spectrum activity against Gram-positive and Gram-negative organisms including anaerobes. Imipenem is partly inactivated in the kidney and this is blocked by cilastin. It should be used with caution in renal failure as it may cause fits.

\section{Meropenem}

It is a bactericidal broad-spectrum carbapenem antibiotic that inhibits cell-wall synthesis. It is effective against most Grampositive and Gram-negative bacteria including Pseudomonas. It has slightly increased activity against Gram-negative species and slightly decreased activity against staphylococci and streptococci compared with imipenem. It is also useful against bacteria with extended spectrum beta lactamases (ESBLs). Meropenem is given intravenously and has less 
frequently caused central nervous system side-effects, including fits, compared with imipenem.

\section{Ertapenem}

This is a carbapenem given once daily and is useful against Gram-positive and Gram-negative organisms and also anaerobes. Bactericidal activity results from inhibition of cell wall synthesis and is mediated through ertapenem binding to penicillin binding proteins. It is stable against hydrolysis by various beta-lactamases, including penicillinases, cephalosporinases and extended spectrum beta-lactamases. It is not active against Pseudomonas, enterococci or against Acinetobacter. It is useful against bacteria with ESBLs and AmpC-producing Gram-negative bacteria. It is generally given to adults as a $1 \mathrm{~g}$ dose, once a day, by intravenous infusion or intramuscular injection. It may be given intramuscularly as $1 \mathrm{~g}$ diluted with $3.2 \mathrm{~mL}$ of $1 \%$ lidocaine.

In a recent study ertapenem was shown to be equivalent in action with piperacillin/tazobactam in treating infected diabetic feet. ${ }^{26}$ In the SIDESTEP study, 586 patients were randomized into two treatment groups to receive intravenously either ertapenem 1 g once daily $(n=295)$ or piperacillin/tazobactam 3.375 g every 6 hours $(n=291)$ for a minimum of 5 days with the option to switch to oral amoxicillin/clavulanate for a total of 5 to 28 days of treatment (parenteral and oral). Patients were assessed by their clinical response between treatment groups at the 10-day post therapy follow-up visit. All patients were eligible to receive appropriate adjunctive treatment methods, such as debridement, and most patients received these treatments. Investigators had the option to add open-label vancomycin if enterococci or MRSA were among the pathogens isolated or if patients had a history of MRSA infection and additional therapy was indicated in the opinion of the investigator. Of those patients described as evaluable (ertapenem $\mathrm{n}=204$; piperacillin/ tazobactam $n=202$ ), $75.0 \%$ of the patients taking ertapenem had a favorable clinical response compared to $70.8 \%$ of the patients taking piperacillin/tazobactam $(\mathrm{CI}=95 \%)$. Rates of favorable microbiological responses and adverse events were also similar between the two treatment groups.

An economic analysis of treatment of diabetic foot infections showed that, compared with piperacillin-tazobactam given 4 times daily iv, ertapenem given once daily was associated with lower drug acquisition and supply costs and less time and labor were devoted to preparation and administration of iv therapy. ${ }^{35} \mathrm{~A}$ cost-minimization analysis was conducted on the drug-dosing data of the subset of patients enrolled in the double-blind randomized trial who were treated solely as inpatients and were clinically evaluable at final assessment $(n=99)$. Cost per dose was calculated from (a) average hospital acquisition price per dose for ertapenem (US\$40.52) or piperacillin/tazobactam (US\$13.58), (b) average US wages and benefits for labor, based on 9 published timeand-motion studies of iv antibiotic preparation and administration (US\$3.10), and (c) consumable supplies, using a $40 \%$ discount off the manufacturer list price (US\$2.90). For each patient, the actual number of antibiotic doses given was multiplied by total cost per dose. There were no significant differences between antibiotic groups with respect to patient demographics, percentage with a severe wound, and mean days of iv therapy. Compared with piperacillin/ tazobactam, patients treated with ertapenem received significantly fewer mean doses $(25.5$ vs $7.5 ; P<0.0001)$ and lower antibiotic-related costs (US\$502.76 vs US\$355.55, respectively; $P<0.001)$. The US\$147.21 difference between groups accounts for approximately $3 \%$ of total hospital Medicare reimbursements for these infections. Compared with piperacillin/tazobactam given 4 times daily iv, ertapenem given once daily iv was associated with lower drug acquisition and supply costs and less time and labor devoted to preparation and administration of iv therapy.

A further study quantified the penetration of ertapenem into bone and synovial tissue. In an open-label study eighteen patients who were undergoing elective total hip replacement received a single, parenteral, $1 \mathrm{~g}$ dose of ertapenem. ${ }^{36}$ One serum, 1 cortical and cancellous bone and 1 synovial tissue sample was collected per patient. The median (interquartile range, IQR) serum concentrations of ertapenem were 70.1 (56.1 to 75.9 ), 10.0 (9.1 to 11.2 ) and $2.6 \mathrm{mg} / \mathrm{L}$ (2.3 to 3.0 ), respectively, at the different time points. The median (IQR) cancellous bone tissue concentrations were 13.2 (10.2 to 14.8), 1.9 (1.7 to 2.1 ) and $0.6 \mu \mathrm{g} / \mathrm{g}(0.4$ to 0.6$)$ at the different time points, corresponding to a median (IQR) tissue/serum penetration ratio of 0.19 ( 0.18 to 0.23$)$. The median (IQR) cortical bone tissue concentrations were 8.0 (6.5 to 9.5), $1.3(1.2$ to 1.3$)$ and $0.3 \mu \mathrm{g} / \mathrm{g}(0.3$ to 0.4$)$ at the different time points, corresponding to a median (IQR) tissue/serum penetration ratio of 0.13 (0.12 to 0.14$)$. The median (IQR) synovial tissue concentrations were $26.2 \mu \mathrm{g} / \mathrm{g}$ (22.7 to 28.4 ), $4.0 \mathrm{mg} / \mathrm{L}(3.7$ to 4.4 ) and $1.0 \mathrm{mg} / \mathrm{L}$ (0.9 to 1.2$)$ at the different time points, corresponding to a median (IQR) tissue/serum penetration ratio of 0.41 ( 0.39 to 0.42$)$. Thus the concentrations after an ertapenem $1 \mathrm{~g}$ dose achieved in cancellous and cortical bone tissue and in synovial tissue were greater than the minimum inhibitory concentrations (MIC) 90 for most aerobic organisms for 24 hours, and for 12 to 24 hours for 
anaerobic bacteria in healthy volunteers undergoing total hip replacement.

For localized organ or tissue infections, drug concentrations in the interstitial space rather than in serum determine the clinical outcome of antimicrobial therapy. One study investigated ertapenem penetration into suction-induced skin blister fluids in 12 healthy young volunteers. ${ }^{37}$ Drug concentrations in skin blister fluids exceeded $4 \mathrm{mg} / \mathrm{L}$ (the MIC at which $90 \%$ of isolates tested are eliminated) throughout the entire dosing interval of 24 hours. The area under the concentrationtime curve for 0 - to 24-hour ratio of blister fluid to plasma was $61 \%(90 \% \mathrm{CI}, 56,65 \%)$ suggesting good blister penetration. A microdialysis study was conducted to measure free, protein-unbound ertapenem concentrations in muscle and subcutaneous tissue. ${ }^{38}$ Ertapenem concentrations in plasma reached a maximum $\left(\mathrm{C}_{\max }\right)$ of $103.3 \pm 26.3 \mathrm{mg} / \mathrm{L}$, a terminal elimination half-life $\left(\mathrm{t}_{1 / 2}\right)$ of $3.8 \pm 0.6$ hours and an $\mathrm{AUC}_{0-}$ of $359.7 \pm 66.5 \mathrm{mg} \cdot \mathrm{h} / \mathrm{L}$. Mean peak concentrations of free, protein-unbound ertapenem in interstitial space fluid of skeletal muscle and subcutaneous adipose tissue were much lower $\left(\mathrm{C}_{\max }=6.7 \pm 4.1\right.$ and $4.0 \pm 1.6 \mathrm{mg} / \mathrm{L}$, respectively). This degree of tissue distribution is consistent with high concentration-dependent plasma protein binding of ertapenem (84\% to $96 \%$ ). $\mathrm{AUC}_{0-}$ values for both muscle and adipose tissue were lower as well $(39.7 \pm 24.8$ and $18.6 \pm 4.6 \mathrm{mg} \cdot \mathrm{h} / \mathrm{L})$. However, unbound interstitial fluid concentrations exceeded MIC90 values for the important skin and skin structure infection (SSSI) pathogens for 7 (subcutis) and 10 hours (muscle) after dosing. Penetration of ertapenem into skeletal muscle and subcutaneous adipose tissue in healthy volunteers measured by in vivo microdialysis was thus satisfactory.

\section{Tigecycline}

As well as its usefulness in infections caused by Grampositive organisms, including MRSA, S. aureus, vancomycinresistant enterococci and streptococci, it is also active against Gram-negative organisms including ESBLs and anaerobes including $B$. fragilis. Strains of Proteus spp. and P. eruginosa may be resistant. Nausea and vomiting may occur particularly as side-effects.

\section{Aminoglycosides}

These include amikacin, gentamicin, netilmicin and tobramycin. Gentamicin is the aminoglycoside of choice in the UK. It is active against some Gram-positive organisms and many Gram-negative organisms. Important side-effects are ototoxicity and nephrotoxicity. These side-effects are dose-related and thus extreme care should be taken with dosage. Gentamicin should be administered with strict blood level monitoring and the trough level should be less than $1 \mathrm{mg} / \mathrm{L}$. The objective of aminoglycoside treatment is to obtain quickly a high peak serum level as the mode of action of the agents is concentration dependent.

\section{Antibiotics used against anaerobic organisms}

Metronidazole is useful against anaerobic bacteria. Patients must be warned not to take alcohol. Clindamycin and co-amoxiclav (amoxicillin/clavulanic acid) also have antianaerobic activity. Meropenem, piperacillin/tazobactam and ertapenem are also active against anaerobes.

\section{Duration of antibiotic therapy}

Patients with infected wounds require early and careful follow-up observation to ensure that the selected medical and surgical treatment regimens have been appropriate and effective. Antibiotic therapy should be continued until there is evidence that the infection has resolved but not necessarily until a wound has healed. The IDSA guidelines have made the following suggestions for the duration of antibiotic therapy as follows: for mild infections, 1 to 2 weeks usually suffices, but some require an additional 1 to 2 weeks; for moderate and severe infections, usually 2 to 4 weeks is sufficient, depending on the structures involved, the adequacy of debridement, the type of soft-tissue wound cover, and wound vascularity.

\section{Antibiotics in patients with renal or liver impairment}

It is ideal to perform renal and liver function tests before starting antibiotic therapy particularly intravenous therapy. These will provide baseline values and also alert the clinician to select appropriate antibiotics if renal or liver impairment is present. Renal impairment is common in diabetic foot patients, and antibiotics can give rise to problems in diabetic patients with reduced renal function for the following reasons:

- Failure to excrete a drug or its metabolites may lead to toxicity.

- Sensitivity to some drugs is enhanced even if elimination is unimpaired.

- Many side-effects are badly tolerated by people in endstage renal failure.

- Some drugs cease to be effective when renal function is impaired. 
Many of these problems can be avoided by reducing the dose or by using alternative antibiotics. Tables indicating dosage of antibiotics in renal impairment should be consulted. ${ }^{11}$ Nephrotoxic antibiotics include the aminoglycosides. Fucidin, rifampicin and doxycycline should be given with caution to patients with liver imparment and serial liver function tests should be performed.

\section{Surveillance for side-effects of antibiotics}

When prescribing antibiotics, it is important to keep a very close surveillance for side-effects particularly skin rashes and gut reactions.

Dermatological drug reactions are self-limited diseases and therefore, generally treatment is symptomatic. Prompt diagnosis and early withdrawal of all suspect drugs are the most important steps. Severe skin reactions include toxic epidermal necrolysis, Stevens-Johnson syndrome and DRESS syndrome (Drug Rash [or Reaction] with Eosinophilia and Systemic Symptoms). ${ }^{39}$ The symptoms of DRESS syndrome usually begin 1 to 8 weeks after exposure to the offending drug. Classic symptoms are rash, fever, lymphadenopathy and involvement of one or more internal organs. The rash is an erythematous skin eruption often progressing to exfoliative dermatitis. It is treated with steroids but can have a mortality rate of about $10 \%$.

Gut reactions can comprise nausea, vomiting, diarrhea and abdominal pain. If this does occur, it is advisable to stop the antibiotics, at least for a short period, to prevent the development of $C$. difficile colitis. Abdominal pain associated with diarrhoea and a raised white blood cell count and fever suggests clostridium difficile infection. Stools should be sent for culture but therapy should be started immediately with either vancomycin $125 \mathrm{mg}$ qds orally or metronidazole $400 \mathrm{mg}$ tds orally. It is important to note that intravenous vancomycin is not active in the gut against $C$. difficile and neither does intravenous metronidazole have a major effect on this organism. The patient should also be given live yoghurt. Acidophilus tablets can also be given to restore the intestinal bacterial flora. In severe cases of diarrhea, patients may need hospitalization and intravenous fluids. If the patient does not respond to these conservative measures then a surgical opinion as to the necessity for a colectomy should be obtained. Recently, new strains of $C$. difficile appear to be more virulent, with ability to produce greater quantities of toxins. PCR ribotype 027 produces much more of the toxins than most other types because a mutation has knocked out the gene that normally restricts toxin production. It causes a greater proportion of severe disease and appears to have a higher mortality. It also seems to be particularly capable of spreading between patients.

\section{Adjunctive therapies}

Granulocyte colony stimulating factor (G-CSF) specifically enhances neutrophil funbction in diabetes and several investigators have explored its use as an adjunct in the treatmemt of diabetic foot infections. ${ }^{40} \mathrm{~A}$ recent Cochrane review has concluded that adjunctive G-CSF treatment in people with a diabetic foot infection, including infected ulcers, did not appear to increase the likelihood of resolution of infection or healing of the foot ulcer. However, it did seem to reduce the need for surgical interventions, especially amputations, and the duration of hospitalization. The authors concluded that "Clinicians might consider adding G-CSF to the usual treatment of diabetic foot infections, especially in patients with a limb-threatening infection, but it is not clear which patients might benefit." ${ }^{40}$

Systemic hyperbaric oxygen therapy may be considered in an individual with severe infected diabetic foot ulcers with full thickness gangrene or abscess, or with a large infected ulcer that has not healed in over 30 days. ${ }^{41}$ In a systematic review evaluating published clinical evidence of the efficacy of hyperbaric oxygen therapy for wound healing and limb salvage it was noted that for patients with diabetic foot ulcers complicated by surgical infection, hyperbaric oxygen reduced the chances of amputation (odds ratio $0.242,95 \%$ CI 0.137 to 0.428 ) and improved the chance of healing (odds ratio 9.992, $95 \%$ CI 3.972 to 25.132$){ }^{42}$

\section{Predictors of unfavorable outcomes}

To aid clinicians in selecting the appropriate approach for treating patients with diabetic foot infections, a recent study investigated whether any baseline clinical findings predicted an unfavorable clinical outcome. ${ }^{43}$ Using data from a large, prospective treatment trial of diabetic foot infections (SIDESTEP), the association between clinical treatment failure and baseline history, physical and laboratory findings was assessed by univariate and multivariate logistic regression analyses. Among 402 patients clinically evaluable 10 days after completing antibiotic therapy, baseline factors significantly $(P<0.05)$ associated by univariate analysis with treatment failure were "severe" (vs "moderate") University of Texas (UT) wound grade; elevated white blood cell count, C-reactive protein or erythrocyte sedimentation rate; high wound severity score; inpatient treatment; low serum albumin; male sex; and skin temperature of affected foot $>10^{\circ} \mathrm{C}$ above that of unaffected foot. By multivariate 
logistic regression only severe UT wound grade (odds ratio 2.1) and elevated white blood cell count (odds ratio 1.7) remained statistically significant. Clinical failure rates were $46 \%$ for patients with both risk factors compared with $10 \%$ for patients with no risk factors and $16 \%$ to $17 \%$ for patients with one risk factor. Increased white blood cell count and severe UT wound grade at baseline, but not other features, were significant independent and additive risk factors for clinical failure in patients treated for a diabetic foot.

\section{Debridement}

Antibiotics alone may be unable to control infection and it is necessary to decide whether debridement or adjunctive surgery is necessary. ${ }^{44}$ Patients with localized and spreading infection usually undergo outpatient sharp debridement to remove callus and allow drainage. In patients with severe infection, the foot should be urgently assessed as to the necessity for surgical debridement. ${ }^{10}$ Early surgical intervention of the affected site is usually necessary as an integral part of infection management. This may include simple debridement of the soft tissues, wide incision and drainage of the pedal compartments, or open amputation to eliminate extensive areas of infection. Although initial drainage procedures can be done at the bedside for neuropathic patients, most require thorough debridement in the operating room. Infected tissue should be sent for culture after debridement. Even very ill patients should be considered for urgent incision, drainage, and debridement procedures, because their illness is directly attributable to the infection severity.

The definite indications for urgent surgical intervention are:

- A large area of infected sloughy tissue.

- Localized fluctuance and expression of pus.

- Crepitus with gas in the soft tissues on X-ray.

- Blue or purplish discoloration of the skin.

\section{Osteomyelitis}

Classically, the treatment of osteomyelitis is surgical removal of bone. But long-term suppressive antibiotic therapy is also used..$^{45}$ As osteomyelitis is usually associated with an infected ulcer and cellulitis, wide spectrum antibiotics should be initially given. On review, antibiotic selection is guided by the results of cultures. Ideally bone culture should be carried out but this is not always practical especially in ischemic feet. ${ }^{46}$ Bone fragments in the base of the wound may be removed as in "office" debridement and then be sent for culture. It is useful to choose antibiotics with good bone penetration such as sodium fusidate, rifampicin clindamycin and ciprofloxacin.
Combinations of antibiotics have also been used including rifampicin and ofloxacin. Antibiotics should be given for at least 12 weeks. Such therapy is often successful with resolution of cellulitis and healing of the ulcer. ${ }^{47}$ A recent report noted that diabetic foot osteomyelitis was effectively managed with oral antimicrobial therapy with or without limited "office" debridement in most cases. ${ }^{48}$ However, if after 6 months' treatment, it is still possible to probe to bone, then operative resection may be necessary. Although urgent surgery is indicated in some patients, non-surgical management of those without limb-threatening infection is associated with a high rate of apparent remission. ${ }^{49}$

\section{Revascularization}

It is important to explore the possibility of revascularization in the infected neuroischemic foot. Improvement of perfusion will not only help to control infection, but will also promote healing of the wound if operative debridement is necessary. Initially, duplex angiography should be carried out to detect the presence of stenoses or occlusions which then may be amenable to angioplasty or bypass.

\section{Metabolic control}

In severe infections, considerable metabolic decompensation may occur. Full resuscitation is urgently required with intravenous fluids and intravenous insulin sliding scale which is often necessary to achieve good blood glucose control whilst the patient is infected. Critically ill patients who require surgery should usually be stabilized before transfer to the operating theater, although surgery should usually not be delayed for $>48$ hours after presentation to the hospital.

\section{Conclusion}

The development of infection in the diabetic foot constitutes a foot care emergency, which requires urgent referral to a specialized foot-care team. The underlying principle is to detect the bacteria responsible and treat aggressively.

\section{Disclosure}

The author declares no conflicts of interest.

\section{References}

1. Pecoraro RE, Reiber GE, Burgess EM. Pathways to diabetic limb amputation. Basis for prevention. Diabetes Care. 1990;13:513-521.

2. Bakker K, Foster AVM, van Houtoum WH, et al, eds. International Diabetes Federation and International Working Group of the Diabetic Foot. 2005 Time to Act. The Netherlands.

3. Reiber GE. Epidemiology of foot ulcers and amputations in the diabetic foot. In: Bowker JH, Pfeifer MA Eds. Levin and O'Neal's The Diabetic Foot. 6th Ed. Mosby, St. Louis; 2001:13-32. 
4. Lipsky, BA Berendt AR, Gunner Deery H, et al. Diagnosis and treatment of diabetic foot infections. Clin Infect Dis. 2004;39:885-910.

5. Lipsky BA. A report from the international consensus on diagnosing and treating the infected diabetic foot. Diabetes Metab Res Rev. 2004; 20 Suppl 1:S68-S77.

6. Berendt AR, Peters EJ, Bakker K, et al. Diabetic foot osteomyelitis: a progress report on diagnosis and a systematic review of treatment. Diabetes Metab Res Rev. 2008;24 Suppl 1:S145-S161.

7. Berendt AR, Peters EJ, Bakker K, et al. Specific guidelines for treatment of diabetic foot osteomyelitis. Diabetes Metab Res Rev. 2008; 24 Suppl 1:S190-S191.

8. Lipsky BA. New developments in diagnosing and treating diabetic foot infections. Diabetes Metab Res Rev. 2008;24 Suppl 1:S66-S71.

9. Lavery LA, Armstrong DG, Murdoch DP, et al. Validation of the infectious diseases society of America's diabetic foot infection classification system. Clin Infect Dis. 2007;44:562-565.

10. Frykberg RG, Zgonis T, Armstrong DG, et al. Diabetic foot disorders: a clinical practice guideline. J Foot Ankle Surg. 2006;45:S2-S66.

11. Edmonds M, Foster A, Sanders L. Practical Manual of Diabetic Foot Care. 2nd ed. Oxford: Blackwell; 2008.

12. Citron DM, Goldstein EJ, Merriam CV, et al. Bacteriology of moderateto-severe diabetic foot infections and in vitro activity of antimicrobial agents. J Clin Microbiol. 2007;45:2819-2828.

13. Dang CN, Prasad YDM, Boulton AJM, et al. Methicillin-resistant Staphylococcus aureus in the diabetic foot clinic: a worsening problem. Diabet Med. 2003;20(2)159-163.

14. Tentolouris N, Petrikkos G, Vallianou N, et al. Prevalence of methicillinresistant Staphylococcus aureus in infected and uninfected diabetic foot ulcers. Clin Microbiol Infect. 2006;12:186-189.

15. Ferry T, Etienne J. Community acquired MRSA in Europe. BMJ. 2007;335:947-948.

16. Lentino JR, Narita M, Yu VL. New antimicrobial agents as therapy for resistant gram-positive cocci. Eur J Clin Microbiol Infect Dis. 2008;27:3-15.

17. Lipsky BA, Itani K, Norden C; Linezolid Diabetic Foot Infections Study Group. Treating foot infections in diabetic patients: a randomized, multicenter, open-label trial of linezolid vs ampicillin-sulbactam/ amoxicillin-clavulanate. Clin Infect Dis. 2004;38:17-24.

18. Stein GE, Schooley S, Peloquin CA, et al. Linezolid tissue penetration and serum activity against strains of methicillin-resistant Staphylococcus aureus with reduced vancomycin susceptibility in diabetic patients with foot infections. Antimicrob Chemother. 2007;60: 819-823.

19. Lipsky BA, Stoutenburgh U, Lipsky BA, et al. Daptomycin for treating infected diabetic foot ulcers: evidence from a randomized, controlled trial comparing daptomycin with vancomycin or semi-synthetic penicillins for complicated skin and skin-structure infections. $J$ Antimicrob Chemother. 2005;55:240-245.

20. Legout M, Assal P, Rohner P, et al. Severe Streptococcus agalactiae infection of the diabetic foot. A deleterious role of Streptococcus agalactiae. Presse Med. 2005;34:491-494.

21. Nelson EA, O'Meara S, Golder, et al. Systematic review of antimicrobial treatments for diabetic foot ulcers. Diabet Med. 2006;23: 348-359.

22. Nelson EA, O’Meara S, Craig D, et al. A series of systematic reviews to inform a decision analysis for sampling and treating infected diabetic foot ulcers. Health Technol Assess. 2006;10:iii-iv, ix-x, 1-221.

23. Lipsky BA. Empirical therapy for diabetic foot infections: are there clinical clues to guide antibiotic selection? Clin Microbiol Infect. 2007; 13:351-353.

24. Bader MS. Diabetic foot infection. Am Fam Physician. 2008;78: 71-79.

25. Clay PG, Graham MR, Lindsey CC, et al. Clinical efficacy, tolerability, and cost savings associated with the use of open-label metronidazole plus ceftriaxone once daily compared with every 6 hours as empiric treatment for diabetic lower-extremity infections in older males. $A m J$ Geriatr Pharmacother. 2004;2:181-189.
26. Lipsky BA, Armstrong DG, Citron DM, et al. Ertapenem vs piperacillin/ tazobactam for diabetic foot infections (SIDESTEP): prospective, randomised, controlled, double-blinded, multicentre trial. Lancet. 2005;366:1695-1703.

27. Weigelt J, Itani K, Stevens D, et al; Linezolid CSSTI Study Group Linezolid vs vancomycin in treatment of complicated skin and soft tissue infections. Antimicrob Agents Chemother. 2005;49:2260-2266.

28. Rybak MJ. The efficacy and safety of daptomycin: first in a new class of antibiotics for Gram-positive bacteria. Clin Microbiol Infect. 2006; 12 Suppl 1:24-32.

29. Eisenstein BI. Lipopeptides, focusing on daptomycin, for the treatment of Gram-positive infections. Expert Opin Investig Drugs. 2004;13: 1159-1169.

30. Davis SL, McKinnon PS, Hall LM, et al. Daptomycin vs vancomycin for complicated skin and skin structure infections: clinical and economic outcomes. Pharmacotherapy. 2003;27(12):1611-1618.

31. Sotto A, Bouziges N, Jourdan N, et al. In vitro activity of tigecycline against strains isolated from diabetic foot ulcers. Pathol Biol (Paris). 2007;55:398-406.

32. Lipsky BA, Giordano P, Choudhri S, et al. Treating diabetic foot infections with sequential intravenous to oral moxifloxacin compared with piperacillin-tazobactam/amoxicillin-clavulanate. J Antimicrob Chemother. 2007;60:370-376.

33. Raymakers JT, Houben AJ, van der Heyden JJ, et al. The effect of diabetes and severe ischaemia on the penetration of ceftazidime into tissues of the limb. Diabet Med. 2001;18:229-234.

34. Harkless L, Boghossian J, Pollak R, et al. An open-label, randomized study comparing efficacy and safety of intravenous piperacillin/ tazobactam and ampicillin/sulbactam for infected diabetic foot ulcers. Surg Infect (Larchmt). 2005;6:27-40.

35. Tice AD, Turpin RS, Hoey CT, et al. Comparative costs of ertapenem and piperacillin-tazobactam in the treatment of diabetic foot infections. Am J Health Syst Pharm. 2007;64:1080-1086.

36. Boselli E, Breilh D, Djabarouti S, Bel JC, Saux MC, et al. Diffusion of ertapenem into bone and synovial tissues. J Antimicrob Chemother. 2007;60:893-896.

37. Laethem T, De Lepeleire I, McCrea J, et al. Tissue penetration by ertapenem, a parenteral carbapenem administered once daily, in suctioninduced skin blister fluid in healthy young volunteers. Antimicrob Agents Chemother. 2003;47:1439-1442.

38. Burkhardt O, Brunner M, Schmidt S, et al. Penetration of ertapenem into skeletal muscle and subcutaneous adipose tissue in healthy volunteers measured by in vivo microdialysis. J Antimicrob Chemother. 2006;58:632-636.

39. Vauthey L, Uçkay I, Abrassart S, et al. Vancomycin-induced DRESS syndrome in a female patient. Pharmacology. 2008;82:138-141.

40. Cruciani M, Lipsky BA, Mengoli C, de Lalla F. Granulocyte-colony stimulating factors as adjunctive therapy for diabetic foot infections. Cochrane Database Syst Rev. 2009; Issue 3. Art. No. CD006810.

41. Hunt D. Foot ulcers and amputations in diabetes. BMJ Clinical Evidence. 2006. URL http://www.clinicalevidence.com/ceweb/conditions/dia/ 0602/0602_I4.jsp.

42. Goldman RJ. Hyperbaric oxygen therapy for wound healing and limb salvage: a systematic review. PMR. 2009;1:471-489.

43. Lipsky BA, Sheehan P, Armstrong DG, et al. Clinical predictors of treatment failure for diabetic foot infections: data from a prospective trial. Int Wound J. 2007;4:30-38.

44. Armstrong DG, Lipsky BA. Diabetic foot infections: stepwise medical and surgical management. Int Wound J. 2004;1:123-132.

45. Senneville E, Lombart A, Beltrand E, et al. Outcome of diabetic foot osteomyelitis treated non-surgically: a retrospective cohort study. Diabetes Care. 2006;(4):637-642.

46. Hartemann-Heurtier A, Senneville E. Diabetic foot osteomyelitis. Diabetes Metab. 2008;34:87-95.

47. Vekatesan P, Lawn S, Macfarlane RM, et al. Conservative management of osteomyelitis in the feet of diabetic patients. Diabet Med. 1997; 14:487-490. 
48. Embil JM, Rose G, Trepman E, et al. Oral antimicrobial therapy for diabetic foot osteomyelitis. Foot Ankle Int. 2006;27:771-779.

49. Game FL, Jeffcoate WJ. Primarily non-surgical management of osteomyelitis of the foot in diabetes. Diabetologia. 2008;51:962-967. Kapur NK, Musunuru K. Clinical efficiency and safety of statins in managing cardiovascular risk. Vasc Health Risk Manag. 2008;4(2): $341-353$.
Meltzer PS, Kallioniemi A, Trent JM. Chromosome alterations in human solid tumors. In: Vogelstein B, Kinzler KW, editors. The Genetic Basis of Human Cancer. New York: McGraw-Hill; 2002:93-113.

\section{Publish your work in this journal}

Vascular Health and Risk Management is an international, peerreviewed journal of therapeutics and risk management, focusing on concise rapid reporting of clinical studies on the processes involved in the maintenance of vascular health; the monitoring, prevention and treatment of vascular disease and its sequelae; and the involvement of metabolic disorders, particularly diabetes. This journal is indexed on PubMed Central and MedLine. The manuscript management system is completely online and includes a very quick and fair peer-review system, which is all easy to use. Visit http://www.dovepress.com/ testimonials.php to read real quotes from published authors.

Submit your manuscript here: http://www.dovepress.com/vascular-health-and-risk-management-journal 